\title{
Technical performance score is associated with outcomes after the Norwood procedure
}

\author{
Meena Nathan, MD, ${ }^{\mathrm{a}}$ Lynn A. Sleeper, $\mathrm{ScD},{ }^{\mathrm{b}}$ Richard G. Ohye, MD, ${ }^{\mathrm{c}}$ Peter C. Frommelt, MD, ${ }^{\mathrm{d}}$ \\ Christopher A. Caldarone, MD, James S. Tweddell, MD, ${ }^{\mathrm{d}}$ Minmin Lu, MS, ${ }^{\mathrm{f}}$ Gail D. Pearson, MD, ScD, ${ }^{\mathrm{g}}$ \\ J. William Gaynor, MD, ${ }^{\mathrm{h}}$ Christian Pizarro, MD ${ }^{\mathrm{i}}$ Ismee A. Williams, MD, MS, ${ }^{\mathrm{j}}$ Steven D. Colan, MD, ${ }^{\mathrm{a}, \mathrm{f}}$ \\ Carolyn Dunbar-Masterson, BSN, RN, ${ }^{\mathrm{a}}$ Peter J. Gruber, MD, ${ }_{\mathrm{k}}^{\mathrm{k}}$ Kevin Hill, MD, Jennifer Hirsch-Romano, MD, ${ }^{\mathrm{c}}$ \\ Jeffrey P. Jacobs, MD, ${ }^{\mathrm{m}}$ Jonathan R. Kaltman, $\mathrm{MD},{ }^{\mathrm{g}} \mathrm{S}$. Ram Kumar, MD, PhD, ${ }^{\mathrm{n}}$ David Morales, MD, \\ Scott M. Bradley, MD, ${ }^{\mathrm{p}}$ Kirk Kanter, MD, ${ }^{\mathrm{q}}$ and Jane W. Newburger, MD, MPH, ${ }^{\mathrm{a}}$ for the Pediatric Heart Network \\ Investigators
}

\begin{abstract}
Objectives: The technical performance score (TPS) has been reported in a single center study to predict the outcomes after congenital cardiac surgery. We sought to determine the association of the TPS with outcomes in patients undergoing the Norwood procedure in the Single Ventricle Reconstruction trial.
\end{abstract}

\begin{abstract}
Methods: We calculated the TPS (class 1, optimal; class 2, adequate; class 3, inadequate) according to the predischarge echocardiograms analyzed in a core laboratory and unplanned reinterventions that occurred before discharge from the Norwood hospitalization. Multivariable regression examined the association of the TPS with interval to first extubation, Norwood length of stay, death or transplantation, unplanned postdischarge reinterventions, and neurodevelopment at 14 months old.
\end{abstract}

Results: Of 549 patients undergoing a Norwood procedure, $356(65 \%)$ had an echocardiogram adequate to assess atrial septal restriction or arch obstruction or an unplanned reintervention, enabling calculation of the TPS. On multivariable regression, adjusting for preoperative variables, a better TPS was an independent predictor of a shorter interval to first extubation $(P=.019)$, better transplant-free survival before Norwood discharge $(P<.001$; odds ratio, 9.1 for inadequate vs optimal), shorter hospital length of stay $(P<.001)$, fewer unplanned reinterventions between Norwood discharge and stage II $(P=.004)$, and a higher Bayley II psychomotor development index at 14 months $(P=.031)$. The TPS was not associated with transplant-free survival after Norwood discharge, unplanned reinterventions after stage II, or the Bayley II mental development index at 14 months.

Conclusions: TPS is an independent predictor of important outcomes after Norwood and could serve as a tool for quality improvement. (J Thorac Cardiovasc Surg 2014;148:2208-14)

See related commentary on pages 2214-5.

Supplemental material is available online.

From the Children's Hospital Boston and Harvard Medical School, ${ }^{\mathrm{a}}$ Boston, Mass; Cytel Inc, ${ }^{\mathrm{b}}$ Cambridge, Mass; University of Michigan Medical School, ${ }^{\mathrm{c}}$ Ann Arbor, Mich; Children's Hospital of Wisconsin and Medical College of Wisconsin, ${ }^{\mathrm{d}}$ Milwaukee, Wis; Hospital for Sick Children, ${ }^{\mathrm{e}}$ Toronto, Ontario, Canada; New England Research Institutes, ${ }^{\mathrm{f}}$ Watertown, Mass; National Heart, Lung, and Blood Institute, ${ }^{g}$ National Institutes of Health, Bethesda, Md; Children's Hospital of Philadelphia and University of Pennsylvania Medical School, ${ }^{\text {h }}$ Philadelphia, Pa; Nemours Cardiac Center, ${ }^{\text {i }}$ Wilmington, Del; Morgan Stanley Children's Hospital of New York-Presbyterian, ${ }^{j}$ New York, NY; University of Iowa Carver College of Medicine, ${ }^{\mathrm{k}}$ Iowa City, Iowa; Duke University, ${ }^{1}$ Chapel Hill, NC; Johns Hopkins All Children's Heart Institute, ${ }^{\mathrm{m}}$ St Petersburg, Fla; Children's Hospital Los Angeles, ${ }^{\mathrm{n}}$ Los Angeles, Calif; Cincinnati Children's Medical Center, ${ }^{\circ}$ Cincinnati, Ohio; Medical University of South Carolina, ${ }^{\mathrm{p}}$ Charleston, SC; and Emory University, ${ }^{\mathrm{q}}$ Atlanta, Ga.

This work was supported by the National Heart, Lung, and Blood Institute (grants HL068269, HL068270, HL068279, HL068281, HL068285, HL068288, HL068290, HL068292, HL085057, HL109737, and HL109781). This work is
The technical performance score (TPS) is a quality improvement tool that was developed to determine the technical adequacy of an intended surgical procedure. It has been validated for a subset of common congenital cardiac operations at a single center. ${ }^{1-8}$ For neonates undergoing the Norwood procedure at a single institution,

solely the responsibility of the authors and does not necessarily represent the official views of the National Heart, Lung, and Blood Institute or National Institutes of Health.

Disclosures: Authors have nothing to disclose with regard to commercial support.

The investigators and centers in the Single Ventricle Reconstruction Trial are listed in Appendix E1.

Presented at The Society of Thoracic Surgeons 50th Annual Meeting, January 25-29, 2014, Orlando, Fla.

Received for publication March 19, 2014; revisions received April 30, 2014; accepted for publication May 29, 2014; available ahead of print July 16, 2014.

Address for reprints: Meena Nathan, MD, Department of Cardiac Surgery, Boston Children's Hospital, Harvard Medical School, 300 Longwood Ave, Boston, MA 02215 (E-mail: meena.nathan@ @ardio.chboston.org).

$0022-5223 / \$ 36.00$

Copyright (c) 2014 by The American Association for Thoracic Surgery http://dx.doi.org/10.1016/j.jtcvs.2014.05.076 

Abbreviations and Acronyms
ASD $=$ atrial septal defect
$\mathrm{IQR}=$ interquartile range
LOS $=$ length of stay
MDI $=$ mental developmental index
$\mathrm{PA}=$ pulmonary artery
PDI $=$ psychomotor developmental index
$\mathrm{SVR}=$ single ventricle reconstruction
TPS $=$ technical performance score

an optimal TPS mitigated the effects of the preoperative physiologic status and illness severity during the initial hospital stay. ${ }^{2,3,9}$ However, the TPS requires validation in a multicenter study.

We sought to determine the validity of TPS across multiple centers using the database from the Pediatric Heart Network's Single Ventricle Reconstruction (SVR) trial. ${ }^{10-15}$ Specifically, we explored whether the TPS could predict early and late outcomes, including resource usage. We hypothesized that the TPS could also identify patients at higher risk of reintervention in the interstage phase.

\section{METHODS}

We performed a secondary analysis of the data from the SVR trial, for which the inclusion and exclusion criteria, study design, and data collection have been previously described. ${ }^{10-15}$

\section{Technical Performance Scoring System}

All subjects were assigned a TPS according to the following data obtained before Norwood hospital discharge: postoperative, protocoldriven transthoracic echocardiographic findings interpreted by a core laboratory and unanticipated surgical or catheter-based reintervention in the areas of Norwood repair before discharge from the hospitalization associated with the Norwood procedure. The TPS module used in our analyses, as modified from the original reported by Bacha and colleagues, ${ }^{2}$ is summarized in Table E1.

In brief, the surgical procedures were divided into components that were assigned a score of class 1 (optimal), class 2 (adequate), or class 3 (inadequate) according to specific echocardiographic criteria and the occurrence of unplanned reinterventions at surgical repair sites before discharge from the Norwood hospitalization (Table E1). The overall classification of the operation as class 1,2 or 3 was determined from the highest class assignment for any of the component subprocedures. All components of the Norwood TPS module in its current version were given equal weight.

Two additional classes were created to allow the inclusion of all subjects in the SVR trial. Class 4 included subjects who had had no core laboratory echocardiograms and no unanticipated reinterventions in the area of Norwood repair before discharge or death. Class 5 included subjects with echocardiograms inadequate for TPS assignment and no unanticipated Norwood surgical reinterventions before discharge or death.

\section{Outcomes}

Our primary outcome was the interval to the initial endotracheal extubation, because it is a well known surrogate for resource usage. We did not choose mortality as our primary endpoint because a vast majority of class 4 (no echocardiogram) patients died and thus could not be assigned a TPS. The secondary outcomes included early mortality or transplantation, defined as occurring before Norwood discharge or within 30 days of the Norwood procedure if discharged before 30 days, whichever was longer; Norwood hospitalization length of stay (LOS); late mortality or transplantation; unplanned reinterventions after Norwood discharge; and the psychomotor development index (PDI) and mental development index (MDI) scores of the "Bayley Scales of Infant Development, 2nd edition."

\section{Classification of Unplanned Reinterventions After Norwood Discharge}

The following postdischarge reinterventions were considered to be attributable to the Norwood procedure technique: (1) any reintervention on the aortic arch, atrial septum, or ascending aorta or proximal pulmonary artery (PA) connection; (2) extensive PA rehabilitation, excluding simple PA augmentation at the stage II or Fontan procedure; and (3) any reintervention on the modified Blalock-Taussig shunt or right ventricle-to-PA shunt between Norwood discharge and the stage II procedure.

The following postdischarge reinterventions were considered not to be attributable to the Norwood procedure technique: (1) coiling of the aortopulmonary or venovenous collaterals; (2) an uncomplicated stage II procedure or Fontan procedure; and (3) any intervention on the superior vena cava after the stage II procedure or Fontan connections after the Fontan procedure.

\section{Statistical Analysis}

The distributions of patient and procedural characteristics by TPS class were compared using the chi-square or Fisher exact test for categorical variables and the Kruskal-Wallis test or 1-way analysis of variance for continuous variables.

Cox regression modeling identified the factors associated with the interval to the initial extubation. The factors associated on univariate analysis at the 0.20 level were candidate predictors for stepwise multivariable modeling. Our multivariable analyses included only the patient factors and preoperative medical variables, because the intraoperative and postoperative outcomes were in the causal pathway of the measures used to calculate the TPS. The TPS was then added to the multivariable model to assess whether it was an independent predictor. Kaplan-Meier estimation with the log-rank test described the association of the interval to initial extubation and the TPS. The same approach was applied to the secondary outcome variables, using linear regression for continuous variables and logistic regression for dichotomous outcomes. We assessed the reliability of the TPS as an independent predictor of each outcome by creating 1000 samples with bootstrapping to determine the percentage of samples in which the TPS was significant at the 0.05 level, conditional on the demographic and preoperative variables already in the multivariable model. A reliability of $\geq 50 \%$ was set as the criterion for retaining the TPS term as an independent predictor.

We assessed whether the 3 components of the TPS (distal arch gradient, atrial septal defect [ASD] gradient, and unplanned reinterventions before Norwood discharge) were associated with Norwood hospitalization LOS, early mortality or transplantation, and reintervention before the stage II procedure. For the echocardiographic components, the medium and high categories for the distal arch gradient and the mean ASD gradient were combined before modeling owing to sparse data.

Sensitivity analyses were also conducted, using the data from subjects with missing and incomplete echocardiograms (class 4 and 5, respectively). The TPS was assumed to be optimal for all, and then inadequate for all, to set the bounds on the relationships between the interval to initial extubation and technical performance.

All analyses were performed using Statistical Analysis Systems, version 9.3 (SAS Institute, Cary, NC).

\section{RESULTS}

The SVR trial randomized 555 subjects, of whom 5 did not undergo a Norwood procedure and 1 withdrew in the 

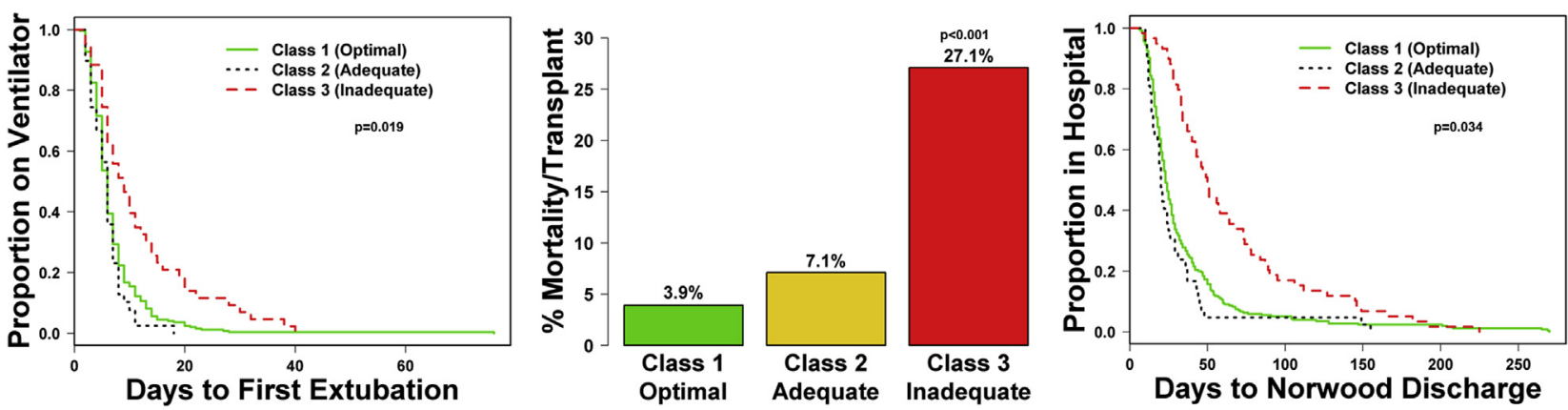

FIGURE 1. Interval to first extubation, early mortality, and Norwood hospital length of stay stratified by technical performance score class. Class 3 subjects had a significantly longer interval to first extubation, greater early mortality, and longer Norwood hospital length of stay.

first week. Of the remaining 549 subjects, $485(88 \%)$ had postoperative echocardiograms adequate for calculating the TPS, and $452(82 \%)$ survived transplant-free to Norwood discharge. Protocol-driven echocardiograms were performed at a median of 14 days (interquartile range [IQR], 8-21) postoperatively. A TPS could be assigned to 356 subjects $(65 \%)$ using the echocardiograms or because of unanticipated reinterventions in the areas of Norwood repair. An additional 56 subjects $(10 \%)$ were class 4 and $137(25 \%)$ class 5 . The baseline characteristics are listed in Table E2. The subjects in class 4 (no echocardiogram) had the lowest birth weight and the highest prevalence of preterm birth and percentage of unknown genetic status. No difference was found in the patient factors between those in class 5 and those with an assigned TPS (Table E3). Among the subjects with a TPS, the distribution was class 1 for $72 \%$, class 2 for $12 \%$, and class 3 for $17 \%$.

\section{Interval to First Extubation}

Of the 452 subjects $(82 \%)$ discharged after the Norwood procedure alive without transplantation, those with TPS class 3 underwent extubation later than those in class 1 or 2 (log-rank test, $P<.001$; Figure 1 ), with a median interval to extubation of 9 days (IQR 5-15), 6 days (IQR, 4-8), and 6 days (IQR, 3-7), respectively. After adjusting for center, the variables independently associated with a longer interval to first extubation included lower gestational age, Hispanic ethnicity, and the presence of a genetic syndrome or anomalies (model $\mathrm{R}^{2}=0.21$ ). When added to this model, TPS was an independent predictor (model $\mathrm{R}^{2}=0.23$, $P=.019,76 \%$ reliability). Class 3 subjects had a longer interval to first extubation (Table 1).

\section{Early Mortality or Transplantation}

Three pre-Norwood characteristics were independently associated with early mortality or transplantation: obstructed pulmonary venous return, cardiac or other surgery before the Norwood operation, and genetic syndrome or anomalies. When added to this model, TPS was an independent risk factor $(P<.001,98 \%$ reliability).
Class 3 subjects had greater odds of early mortality or transplantation, and class 1 and 2 subjects had similar risks of early mortality or transplantation (Table E4 and Figure 1). The $\mathrm{R}^{2}$ increased from 0.14 to 0.26 (Table 2).

\section{Norwood Hospital LOS in Transplant-Free Survivors}

The median LOS $(\mathrm{n}=329)$ was significantly longer in those in class 3 than in those in class 1 and 2 (median 50 vs 23 and 20 days, respectively; Figure 1). The TPS was an independent predictor of the Norwood LOS $(P<.001,100 \%$ reliability) when added to the multivariable model that included center and genetic syndrome or anomalies. The $\mathrm{R}^{2}$ increased from 0.15 to 0.20 (Table 3).

\section{Late Reinterventions Attributable to the Norwood}

The data were analyzed in 2 periods: after Norwood discharge but before stage II and after the stage II procedure. Of the 329 subjects with a TPS class, including $37(11 \%)$ who died between Norwood discharge and stage II surgery, those in class 1 were the least likely to have required reintervention between Norwood discharge and stage II (logistic regression, $P=.003 ; 78 \%$ reliability; Table 4 and Table E5). The TPS was not associated with late reinterventions from the stage II operation to age 12 months.

\section{Mortality or Transplantation After Norwood Discharge}

Of the 448 transplant-free survivors to Norwood discharge, 96 had died $(n=85)$ or underwent transplantation $(\mathrm{n}=11)$ by 3 years after randomization. The independent predictors of a greater hazard of death or transplantation between Norwood discharge and 3 years after randomization included preterm delivery, obstructed pulmonary venous return, and greatest pre-Norwood lactate level. When added to this model, the TPS was not an independent predictor (Tables E6 and E7).

\section{Neurodevelopmental Outcomes}

The distributions of the PDI and MDI scores are presented in Figure E1. The independent preoperative 
TABLE 1. Multivariable Cox regression model for interval to first extubation* $\left(\mathbf{n}=324, \mathbf{R}^{2}=\mathbf{0 . 2 3}\right)$

\begin{tabular}{|c|c|c|c|}
\hline Variable & HR & $95 \% \mathbf{C I}$ & $P$ value \\
\hline Center & & & $<.001$ \\
\hline Gestational age (mo) & 0.88 & $0.82-0.95$ & $<.001$ \\
\hline Hispanic & & & .03 \\
\hline Yes & 1.40 & $1.04-1.90$ & \\
\hline No & Reference & & \\
\hline Genetic syndrome or other anomalies & & & .03 \\
\hline Yes & 1.51 & $1.12-2.06$ & \\
\hline No & Reference & & \\
\hline Unknown & 1.19 & $0.84-1.66$ & \\
\hline TPS & & & .02 \\
\hline Class 1 (optimal) & 0.59 & $0.41-0.85$ & \\
\hline Class 2 (adequate) & 0.62 & $0.38-1.00$ & \\
\hline Class 3 (inadequate) & Reference & & \\
\hline
\end{tabular}

predictors of a lower PDI score were center, lower birth weight, and genetic syndrome or anomalies. When the TPS was added to the model, a worse TPS class was associated with a lower PDI score $(P=.031,66 \%$ reliability), with the model $\mathrm{R}^{2}$ increasing from 0.19 to 0.22 . A lower MDI score was independently associated with center, lower birth weight, lower Apgar score at 1 minute, intubation for respiratory failure or metabolic acidosis, and genetic syndrome or anomalies. When added to this model, the TPS class was not associated with the MDI score.

\section{TPS Components}

We analyzed the 3 components of the TPS available in the SVR trial database (distal arch gradient, ASD gradient, and unplanned predischarge reintervention in the areas of Norwood repair) to test their associations with the selected outcomes. Unplanned predischarge reinterventions in the areas of Norwood repair could occur in any component of the Norwood TPS. The reinterventions that resulted in class

TABLE 2. Multivariable logistic regression model for early death or transplantation $\left(\mathrm{n}=356, \mathrm{R}^{2}=\mathbf{0 . 2 6}\right)$

\begin{tabular}{lccc}
\hline \multicolumn{1}{c}{ Variable } & OR & $\mathbf{9 5} \%$ CI & $\boldsymbol{P}$ value \\
\hline $\begin{array}{l}\text { Pre-Norwood intubation } \\
\text { Genetic syndrome or other anomalies }\end{array}$ & 2.38 & $1.01-5.61$ & .05 \\
$\quad$ & 0.83 & $0.23-3.01$ & .002 \\
$\quad$ Yes & Reference & & \\
$\quad$ No & 4.43 & $1.72-11.4$ & \\
$\quad$ Unknown & & & $<.001$ \\
TPS & 0.11 & $0.04-0.26$ & \\
$\quad$ Class 1 (optimal) & 0.19 & $0.05-0.75$ & \\
Class 2 (adequate) & Reference & & \\
Class 3 (inadequate) & & & \\
All $P$ values were statistically significant. $O R$, Odds ratio; $C I$, confidence interval; \\
TPS, technical performance score.
\end{tabular}

TABLE 3. Multivariable Cox regression model for Norwood length of stay* $\left(\mathbf{n}=329, \mathbf{R}^{2}=\mathbf{0 . 2 0}\right)$

\begin{tabular}{lcc}
\hline \multicolumn{1}{c}{ Variable } & HR $(\mathbf{9 5} \%$ CI $)$ & $\boldsymbol{P}$ value \\
\hline Center & & .01 \\
Genetic syndrome or other anomalies & & .001 \\
$\quad$ Yes & $1.63(1.21-2.19)$ & \\
No & Reference & \\
Unknown & $1.65(1.18-2.30)$ & \\
TPS & $0.44(0.31,0.63)$ & \\
Class 1 (optimal) & $0.35(0.22,0.56)$ & \\
Class 2 (adequate) & Reference & \\
Class 3 (inadequate) & & .001 \\
Pre-Norwood complication & $1.33(1.02-1.72)$ & \\
Yes & Reference & \\
No &
\end{tabular}

All $P$ values were statistically significant. $H R$, Hazard ratio; $C I$, confidence intervals; TPS, technical performance score. *Excluding data of subjects who died or underwent cardiac transplant during the hospitalization.

3 TPS assignment are listed in Table E8, and the echocardiographic findings in the 59 subjects assigned to class 3 are summarized in Table E9. In multivariable analysis, pre-Norwood discharge reintervention was significantly associated with a greater early mortality or transplantation rate (odds ratio, 8.36; 95\% confidence interval, 3.54-19.7; $P<.001)$ and longer median Norwood LOS (51 days, IQR, 33-86; vs 23 days, IQR, 16-36; $P<.01)$. A distal arch gradient of $\geq 20 \mathrm{~mm} \mathrm{Hg}$ was significantly associated with a greater odds of prestage II reintervention (odds ratio, 3.28, 95\% confidence interval, 1.59-6.76; $P<.001)$. The ASD gradient was not associated with these 3 outcomes.

\section{Sensitivity Analyses}

To assess the effect of the exclusion of class 5 (incomplete echocardiograms) on the interval to first extubation, we included 137 class 5 subjects in the Cox regression analyses under 2 extreme assumptions: that all were class 1 and that all were class 3 . When all class 5 subjects were assumed be class 1 , the TPS remained a

TABLE 4. Multivariable logistic regression model for prestage II reintervention $\left(n=318, R^{2}=0.12\right)$

\begin{tabular}{lccc}
\hline \multicolumn{1}{c}{ Variable } & OR & $\mathbf{9 5} \% \mathbf{C I}$ & $\boldsymbol{P}$ value \\
\hline $\begin{array}{l}\text { Prenatal diagnosis of CHD } \\
\quad \text { Yes }\end{array}$ & 2.77 & $1.24-6.18$ & .01 \\
$\quad$ No & Reference & & \\
SES score & 1.06 & $1.01-1.12$ & .03 \\
TPS & & & .01 \\
$\quad$ Class 1 (optimal) & Reference & & \\
Class 2 (adequate) & 2.93 & $1.41-6.11$ & \\
Class 3 (inadequate) & 2.02 & $0.94-4.32$ & \\
\hline
\end{tabular}

All $P$ values were statistically significant. $O R$, Odds ratio; $C I$, confidence interval; $C H D$, congenital heart disease; SES, socioeconomic status; TPS, technical performance score. *Class 2 versus 3 did not differ $(P=.681)$. 
significant $(P<.001)$ independent predictor. In contrast, when all class 5 subjects were presumed to be class 3 , the TPS was no longer associated $(P=.99)$ with the interval to first extubation. However, because the characteristics of the subjects with an incomplete echocardiogram were similar to those with an assignable TPS, this latter sensitivity assumption (100\% class 3$)$ was discordant with the observed percentage of subjects with a score who were assigned to class $3(17 \%)$.

We did not perform a sensitivity analysis regarding the interval to first extubation for class 4 (no echocardiogram), because only 2 of the 56 class 4 subjects survived to Norwood discharge and had interval-to-extubation data. Instead, we performed a sensitivity analysis using the regression model for early mortality or transplantation and included the class 4 subjects, assuming that all had either class 1 or 3 TPS. When class 4 subjects were all assumed to be class 1 , the TPS remained associated $(P=.050)$ with early mortality or transplantation. However, we would expect that class 4 subjects would be most likely to have had an inadequate TPS (class 3 ) rather than an optimal TPS (class 1), because $96 \%$ of the class 4 subjects died or underwent transplantation. When the class 4 subjects were all assigned to class 3 , the TPS was strongly associated with mortality $(P<.001)$.

\section{DISCUSSION}

Although the role of human factors in the outcomes after surgery has been well described, ${ }^{16-18}$ the technical adequacy of the repair could still be the single most important factor in determining the outcomes. This premise has been proved in other surgical fields ${ }^{19-21}$ in which assessment models have been based on methods such as video recordings, and models of surgical skills assessment have been incorporated into surgical training programs. ${ }^{22-25}$ However, a tool using routine clinical information for measurement of technical adequacy has not been validated. As previously described at a single center, $^{3-9}$ the TPS is based on information available from routine clinical care, including postoperative echocardiographic findings and reintervention in the anatomic areas of surgical repair. Moreover, the TPS has been associated with early outcomes, such as mortality, major adverse events, longer LOS, and higher costs, and mid-term outcomes after hospital discharge, such as transplant-free survival and unplanned reinterventions.

In the present multicenter study, we found that the TPS was an independent predictor of both early and mid-term outcomes after the Norwood procedure. Specifically, our multivariable model, which included baseline patient factors and preoperative variables, found that a worse TPS was significantly associated with a longer interval to first extubation, greater early mortality or transplantation, a longer Norwood LOS, more unanticipated interventions between Norwood discharge and the stage II procedure, and lower PDI scores at 14 months of age. We did not find an association between the TPS and unanticipated reinterventions after the stage II procedure, the 14-month MDI score, or late mortality or transplantation. The association of a worse TPS with a lower 14-month PDI score but not MDI score is consistent with the nearly universal observation that the PDI scores are more affected than the MDI scores in children with congenital heart disease. ${ }^{26-29}$ Early death in subjects with inadequate repair might explain the lack of an association of the TPS with late mortality or transplantation and the greater rate of reintervention before stage II among the subjects in class 2 compared with class 3. Analyses of the components of the TPS showed that reinterventions were significantly associated with early mortality and Norwood LOS, and higher arch gradients were associated with prestage II reinterventions. The present multicenter study has validated the generalizability of the TPS across centers that vary widely in geographic location and volume.

The effect of the TPS on Norwood outcomes is relatively modest compared with that of the other independent risk factors measured in the SVR trial. This was, in part, because the regression models in the present study could not include the intraoperative and postoperative variables that are in the causal pathway of the factors on which calculation of the TPS is based. For example, in an earlier report from the SVR trial, ${ }^{30}$ extracorporeal membrane oxygenation and an open sternum on the day of the Norwood procedure were among the most powerful independent predictors of 30-day and hospital mortality and the interval to first extubation after the Norwood procedure, the primary outcome in our study. Similarly, the independent predictors of interstage mortality included the use of a modified Blalock-Taussig shunt rather than a right ventricular-to-PA shunt in subjects with no or mild postoperative atrioventricular valve regurgitation and greater number of post-Norwood complications. ${ }^{31}$ Our regression models did, however, consider patient and preoperative medical risk factors previously shown to be independent risk factors of adverse outcomes before Norwood discharge and in the interstage, such as low birthweight, ${ }^{30}$ lower gestational age, ${ }^{31}$ and anatomic factors $^{31}$ (eg, the presence of aortic atresia or mitral atresia). The TPS is among the most modifiable of the various risk factors demonstrated in the SVR trial.

The TPS has been based on the transthoracic echocardiogram performed before hospital discharge. A scoring system for intraoperative echocardiography might provide timely information and elucidate components of an "inadequate" procedure that would benefit from immediate intraoperative revision. Recent work has shown that intraoperative revision of residual lesions improves in-hospital outcomes. ${ }^{5}$ 
Our analysis should be viewed in light of its limitations. We were unable to calculate the TPS for one third of the subjects because of missing or incomplete protocol echocardiograms, together with the absence of reinterventions at the sites of initial surgical correction. Mortality was a secondary study outcome, because its analysis with respect to TPS was conditional on early survival (ie, the vast majority of subjects without echocardiograms [91\%] died early-before the protocol echocardiogram was performed [class 4 subjects]). Although it seems likely that most of these subjects would have had a class 3 TPS, our study design did not allow us to test this hypothesis directly. In the sensitivity analyses, assuming that all these subjects had either class 1 or 3 TPS, the TPS remained an independent predictor of mortality before Norwood discharge. The primary endpoint of the present study, the interval to initial extubation, might have been affected by center practices and protocols. However, the center was considered as a covariate in the multivariable models, and other measures of overall hospital complexity, such as the LOS in the cardiac intensive care unit, would have been even more affected by center variation. ${ }^{32}$ Finally, the components of the TPS were derived by the consensus of experts, rather than by determination of cutpoints from analyses of prospectively collected data, and TPS classes 1 and 2 were often similar in their prediction of the outcomes. A prospective multicenter study of the Pediatric Heart Network, currently in its planning phase, will refine the cutpoints of the TPS using both a multicenter expert panel and an analysis of prospectively collected data.

\section{CONCLUSIONS}

The TPS is an independent predictor of both early and mid-term outcomes in patients with hypoplastic left heart syndrome and other single right ventricle anomalies undergoing the Norwood procedure. With additional multicenter refinement and testing, the TPS could be used as a tool for quality improvement, with the potential for impact, not only on patient outcomes, but also on resource usage in this costly, high-risk population.

\section{References}

1. Larrazabal LA, del Nido PJ, Jenkins KJ, Gauvreau K, Lacro S, Colan SD, et al. Measurement of technical performance in congenital heart surgery: a pilot study. Ann Thorac Surg. 2007;83:179-84.

2. Bacha EA, Larrazabal LA, Pigula FA, Gauvreau K, Jenkins KJ, Colan SD, et al. Measurement of technical performance in surgery for congenital heart disease: the stage I Norwood procedure. J Thorac Cardiovasc Surg. 2008; 136:993-7.

3. Karamichalis JM, del Nido PJ, Thiagarajan RR, Liu H, Gauvreau K, Pigula FA, et al. Early postoperative severity of illness predicts outcomes following the Stage I Norwood procedure. Ann Thorac Surg. 2011;92:660-5.

4. Nathan M, Karamichalis J, Liu H, del Nido P, Pigula F, Thiagarajan R, et al. Intraoperative adverse events can be compensated in infants after cardiac surgery: a prospective study. J Thorac Cardiovasc Surg. 2011;142:1098-107.

5. Nathan M, Karamichalis J, Liu H, Emani S, Baird C, Pigula F, et al, Surgical technical performance scores are predictors for late mortality and unplanned reinterventions in infants after cardiac surgery. J Thorac Cardiovasc Surg. 2012;144:1095-101.

6. Nathan M, Pigula FA, Colan S, Liu H, Mayer JE, Fynn-Thompson F, et al. Inadequate technical performance scores are associated with late mortality and need for late re-intervention in a 13 month cohort of patients followed for 4 years. Ann Thorac Surg. 2013;96:664-9.

7. Nathan M, Karamichalis J, Liu H, Gauvreau K, Colan S, Pigula FA, et al. Technical performance scores are strongly associated with early mortality, postoperative adverse events and ICU length of stay-analysis of consecutive discharges over 2 years. J Thorac Cardiovasc Surg. 2014;147: 389-96.

8. Nathan M, Sadhwani A, Gauvreau K, Ware J, Agus M, Newburger J, et al. Association between technical performance scores and neurodevelopmental outcomes after congenital cardiac surgery. J Thorac Cardiovasc Surg. 2014; 148:232-7.

9. Karamichalis JM, Thiagarajan RR, Liu H, Mamic P, Gauvreau K, Bacha EA. Stage I Norwood: optimal technical performance improves outcomes irrespective of preoperative physiologic status or case complexity. J Thorac Cardiovasc Surg. 2010;139:962-8.

10. Ohye RG, Gaynor JW, Ghanayem NS, Goldberg CS, Laussen PC, Frommelt PC, et al. Design and rationale of a randomized trial comparing the Blalock-Taussig and right ventricle-pulmonary artery shunts in the Norwood procedure. J Thorac Cardiovasc Surg. 2008;136:968-75.

11. Ohye RG, Sleeper LA, Mahony L, Newburger JW, Pearson GD, Lu M, et al Comparison of shunt types in the Norwood procedure for single-ventricle lesions. N Engl J Med. 2010;362:1980-92.

12. Tweddell JS, Sleeper LA, Ohye RG, Williams IA, Mahony L, Pizarro C, et al Intermediate-term mortality and cardiac transplantation in infants with single-ventricle lesions: risk factors and their interaction with shunt type. J Thorac Cardiovasc Surg. 2012;144:152-9.

13. Newburger JW, Sleeper LA, Bellinger DC, Goldberg CS, Tabbutt S, Lu M, et al Early developmental outcome in children with hypoplastic left heart syndrome and related anomalies: the single ventricle reconstruction trial. Circulation 2012;125:2081-91.

14. Atz AM, Travison TG, Williams IA, Pearson GD, Laussen PC, Mahle WT, et al. Prenatal diagnosis and risk factors for preoperative death in neonates with single right ventricle and systemic outflow obstruction: screening data from the Pediatric Heart Network Single Ventricle Reconstruction trial. J Thorac Cardiovasc Surg. 2010;140:1245-50.

15. Virzi L, Pemberton V, Ohye RG, Tabbutt S, Lu M, Atz TC, et al. Reporting adverse events in a surgical trial for complex congenital heart disease: the Pediatric Heart Network experience. J Thorac Cardiovasc Surg. 2011;142:531-7.

16. de Leval MR, Carthey J, Wright DJ, Farewell VT, Reason JT. Human factors and cardiac surgery: a multicenter study. J Thorac Cardiovasc Surg. 2000;119:661-72.

17. Barach P, Johnson JK, Ahmad A, Galvan C, Bognar A, Duncan R, et al. A prospective observational study of human factors, adverse events and patient outcomes in surgery for pediatric cardiac disease. J Thorac Cardiovasc Surg. 2008;136:1422-8.

18. Schraagen JM, Schouten T, Smit M, Hass F, van der Beek D, van den Ven J, et al. A prospective study of paediatric cardiac surgical microsystems: assessing the relationships between non routine events, team work and patient outcomes. BMJ Qual Saf. 2011;20:599-603.

19. Birkmeyer JD, Finks JF, O’Reilly A, Oerline M, Carlin AM, Nunn AR, et al Surgical skill and complications after bariatric surgery. N Engl J Med. 2013; 369:1434-42.

20. Zevin B, Bonrath EM, Aggarwal R, Dedy JN, Ahmed N, Grantcharov TP. the ATLAS group. Development, feasibility, validity, and reliability of a scale for objective assessment of operative performance in laparoscopic gastric bypass surgery. J Am Coll Surg. 2013;216:955-65.

21. Carty MJ, Chan R, Huckman R, Snow D, Orgill DP. A detailed analysis of the reduction mammaplasty learning curve: a statistical process model for approaching surgical performance improvement. Plast Reconstr Surg. 2009; 124:706-14

22. Martin JA, Regehr G, Reznick R, Macare H, Murnaghan J, Hutchison C, et al. Objective structured assessment of technical skill (OSATS) for surgical residents. Br J Surg. 1997;84:273-8.

23. Macrae HM. Objective assessment of technical skill. Surgeon. 2011;9(suppl 1): S23-5.

24. Gofton WT, Dudek NL, Wood TJ, Balaa F, Hamstra SJ. The Ottawa surgical competency operating room evaluation (O-SCORE): a tool to assess surgical competence. Acad Med. 2012;87:1401-7. 
25. Faurie C, Khadra M. Technical competence in surgeons. ANZ J Surg. 2012;82: 682-90.

26. Mahle WT, Lu M, Ohye RG, Gaynor JW, Goldberg CS, Sleeper LA, et al. A predictive model for neurodevelopmental outcome after the Norwood procedure. Pediatr Cardiol. 2013;34:327-33.

27. Gaynor JW, Gerdes M, Zackai EH, Bernbaum J, Wernovsky G, Clancy RR, et al. Apolipoprotein E genotype and neurodevelopmental sequelae of infant cardiac surgery. J Thorac Cardiovasc Surg. 2003;126:1736-45.

28. Goldberg CS, Bove EL, Devaney EJ, Mollen E, Schwartz E, Tindall S, et al. A randomized clinical trial of regional cerebral perfusion versus deep hypothermic circulatory arrest: outcomes for infants with functional single ventricle. J Thorac Cardiovasc Surg. 2007;133:880-7.

29. Limperopoulos C, Majnemer A, Shevell MI, Rohlicek C, Rosenblatt B, Tchervenkov C, Darwish HZ. Predictors of developmental disabilities after open heart surgery in young children with congenital heart defects. $J$ Pediatr. 2002:141:51-8

30. Tabbutt S, Ghanayem N, Ravishankar C, Sleeper LA, Cooper DS, Frank DU, et al. Risk factors for hospital morbidity and mortality after the Norwood procedure: a report from the Pediatric Heart Network Single Ventricle Reconstruction trial. J Thorac Cardiovasc Surg. 2012;144: 882-95.

31. Ghanayem NS, Allen KR, Tabbutt S, Atz AM, Clabby ML, Cooper DS, et al. Interstage mortality after the Norwood procedure: results of the multicenter single ventricle reconstruction trial. J Thorac Cardiovasc Surg. 2012;144: 896-906.

32. Pasqali SK, Ohye RG, Lu M, Kaltman J, Caldarone CA, Pizarro C, et al. Variation in perioperative care across centers for infants undergoing the Norwood procedure. J Thorac Cardiovasc Surg. 2012;144:915-21.

\title{
EDITORIAL COMMENTARY
}

\section{Utility of the technical performance score for the Norwood operation ... every score should know its limitations}

\author{
Tara Karamlou, MD
}

See related article on pages 2208-14.

The article by Nathan and colleagues ${ }^{1}$ in this issue of the Journal provides additional data regarding the relationship of the technical performance score (TPS) to outcomes after the Norwood procedure among participants of the Single Ventricle Reconstruction Trial (SVR). Nathan and colleagues ${ }^{1}$ conducted an ad hoc analysis of the SVR cohort of neonates undergoing the Norwood operation. By means of discharge echocardiography, available for $365(65 \%)$ of the 549 patients in the study, a TPS category of optimal $(72 \%)$, adequate $(12 \%)$, or inadequate $(17 \%)$, was assigned. The components of the TPS, which are weighted equally, include restriction at the atrial septum, residual aortic arch obstruction, and intervention on the chosen source of pulmonary blood flow. Neurodevelopmental outcome was assessed with the Bayley Scales of Infant Development (2nd edition). By means of multivariable regression, Nathan and colleagues ${ }^{1}$ found that better TPS was associated with shorter time to initial extubation, improved transplant-free survival before discharge after the Norwood operation, shorter hospital stay, lower

From the Division of Pediatric Cardiac Surgery, Benioff Children's Hospital, University of California, San Francisco, San Francisco, Calif.

Disclosures: Author has nothing to disclose with regard to commercial support.

Received for publication Sept 12, 2014; revisions received Sept 23, 2014; accepted for publication Sept 27, 2014

Address for reprints: Tara Karamlou, MD, Benioff Children's Hospital, University of prevalence of unplanned interstage interventions, and better Bayley Scales Psychomotor Developmental Index (PDI) subscale scores at 14 months.

I applaud Nathan and colleagues ${ }^{1}$ for extending their considerable work in this area and for using a multi-institutional cohort with prospectively collected data. The TPS has promise as the first objective tool that can both inform care and provide a platform for individual surgeon evaluation. There are important limitations to this study, however, that should temper Nathan and colleagues' conclusions $^{1}$ regarding the impact of the TPS on postNorwood outcomes.

Factors that affect outcomes after the Norwood operation have been studied in detail with both prospective and retrospective data. It is clear from the majority of these studies that surgeon, institution, and patient factors play critical roles; however, the relative contributions of each component remain unknown and vary with different lesions and procedures. In analysis of arguably the best data collection available, ${ }^{2-4}$ surgeon factors in the Norwood operation, including volume and experience (ostensibly a surrogate for technical performance), were identified as having minor influence on mortality relative to center or patient

California, San Francisco, 513 Parnassus Ave, Suite S-549, San Francisco, CA 94143 (E-mail: tara.karamlou@ucsfmedctr.org).

J Thorac Cardiovasc Surg 2014;148:2214-5 $0022-5223 / \$ 36.00$

Copyright $(2014$ by The American Association for Thoracic Surgery http://dx.doi.org/10.1016/j.jtcvs.2014.09.103 


\section{APPENDIX E1. SINGLE VENTRICLE RECONSTRUCTION TRIAL PARTICIPANTS}

National Heart, Lung, and Blood Institute: Gail Pearson, Victoria Pemberton, Rae-Ellen Kavey, Mario Stylianou, Marsha Mathis, Jonathan Kaltman

Network Chairperson: University of Texas Southwestern Medical Center, Lynn Mahony

Data Coordinating Center: New England Research Institutes, Lynn Sleeper (primary investigator [PI]), Sharon Tennstedt (PI), Steven Colan, Lisa Virzi, Patty Connell, Victoria Muratov, Lisa Wruck, Minmin Lu, Dianne Gallagher, Anne Devine, Thomas Travison, David F. Teitel

Core Clinical Site Investigators: Children's Hospital Boston, Jane W. Newburger (PI), Peter Laussen, Pedro del Nido, Roger Breitbart, Jami Levine, Ellen McGrath, Carolyn Dunbar-Masterson; Children's Hospital of New York, Wyman Lai (PI), Beth Printz (currently at Rady Children's Hospital), Daphne Hsu (currently at Montefiore Medical Center), William Hellenbrand (currently at Yale New Haven Medical Center), Ismee Williams, Ashwin Prakash (currently at Children's Hospital Boston), Ralph Mosca (currently at New York University Medical Center), Darlene Servedio, Rozelle Corda, Rosalind Korsin, Mary Nash; Children's Hospital of Philadelphia, Victoria L. Vetter (PI), Sarah Tabbutt (currently at the University of California, San Francisco), J. William Gaynor (study co-chairperson), Chitra Ravishankar, Thomas Spray, Meryl Cohen, Marisa Nolan, Stephanie Piacentino, Sandra DiLullo, Nicole Mirarchi; Cincinnati Children's Medical Center, D. Woodrow Benson (PI), Catherine Dent Krawczeski, Lois Bogenschutz, Teresa Barnard, Michelle Hamstra, Rachel Griffiths, Kathie Hogan, Steven Schwartz (currently at the Hospital for Sick Children, Toronto), David Nelson, David Morales; North Carolina Consortium: Duke University, East Carolina University, Wake Forest University, Page A. W. Anderson (PI; deceased), Jennifer Li (PI), Wesley Covitz, Kari Crawford, Michael Hines, James Jaggers, Theodore Koutlas, Charlie Sang, Jr, Lori Jo Sutton, Mingfen Xu, Kevin Hill; Medical University of South
Carolina, J. Philip Saul (PI), Andrew Atz, Girish Shirali, Scott Bradley, Eric Graham, Teresa Atz, Patricia Infinger; Primary Children's Medical Center and University of Utah, Salt Lake City, Utah, L. LuAnn Minich (PI), John Hawkins (deceased), Michael Puchalski, Richard Williams, Linda Lambert, Jun Porter, Marian Shearrow, Peter Gruber; Hospital for Sick Children, Toronto, Brian McCrindle (PI), Joel Kirsh, Chris Caldarone, Elizabeth Radojewski, Svetlana Khaikin, Susan McIntyre, Nancy Slater; University of Michigan, Caren S. Goldberg (PI), Richard G. Ohye (study chairperson), Cheryl Nowak, Jennifer Hirsch-Romano; Children's Hospital of Wisconsin, Nancy Ghanayem (PI), James Tweddell, Kathy Mussatto, Michele Frommelt, Lisa Young-Borkowski

Auxiliary Sites: Children's Hospital Los Angeles, Alan Lewis (PI), Vaughn Starnes, Nancy Pike, S. Ram Kumar; Johns Hopkins All Children's Heart Institute, Jeffrey P. Jacobs (PI), James A. Quintessenza, Paul J. Chai, David S. Cooper, J. Blaine John, James C. Huhta, Tina Merola, Tracey Cox; Emory University, Kirk Kanter, William Mahle, Joel Bond, Leslie French, Jeryl Huckaby; Nemours Cardiac Center, Christian Pizarro, Carol Prospero, Julie Simons, Gina Baffa; University of Texas Southwestern Medical Center, Ilana Zeltzer (PI), Tia Tortoriello, Deborah McElroy, Deborah Town

Angiography Core Laboratory: Duke University, John Rhodes, J. Curt Fudge

Echocardiography Core Laboratories: Children's Hospital of Wisconsin, Peter Frommelt; Children's Hospital Boston, Gerald Marx

Genetics Core Laboratory: Children's Hospital of Philadelphia, Catherine Stolle

Protocol Review Committee: Michael Artman (chairperson), Erle Austin, Timothy Feltes, Julie Johnson, Thomas Klitzner, Jeffrey Krischer, G. Paul Matherne

Data and Safety Monitoring Board: John Kugler (chairperson), Rae-Ellen Kavey (executive secretary), David J. Driscoll, Mark Galantowicz, Sally A. Hunsberger, Thomas J. Knight, Holly Taylor, Catherine L. Webb 

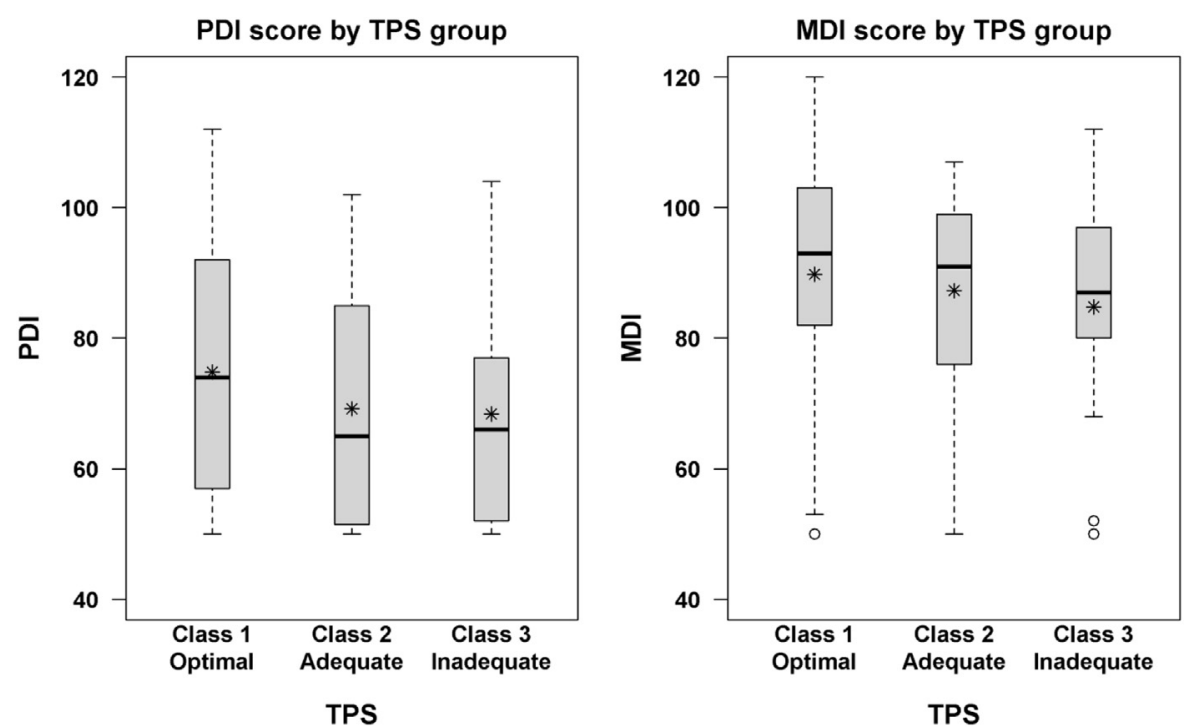

FIGURE E1. Box plots of Bayley psychomotor developmental index (PDI) and mental developmental index (MDI) scores by technical performance score (TPS) class. Those with class 1, optimal TPS, had higher MDI and PDI scores. Asterisk represents the mean, and the bottom, center, and top edges of the box represent the 25th, 50th, and 75th percentiles.

TABLE E1. Modified technical performance score module for Norwood procedure used to assign TPS in the SVR cohort

\begin{tabular}{llll}
\hline Subprocedure & Class 1 (optimal) & Class 2 (adequate) & Class 3 (inadequate) \\
Proximal arch reconstruction & NA & NA & Reintervention \\
Distal arch reconstruction & Peak gradient $<20 \mathrm{~mm} \mathrm{Hg}$ & Peak gradient 20-40 mm Hg & Reintervention or peak gradient $>40 \mathrm{~mm} \mathrm{Hg}$ \\
Coronary perfusion & NA & NA & Reintervention \\
Atrial septectomy & Mean gradient $<2 \mathrm{~mm} \mathrm{Hg}$ & Mean gradient 3-4 mm Hg & Reintervention or mean gradient $>4 \mathrm{~mm} \mathrm{Hg}$ \\
MBTS & Patent & Patent & Reintervention \\
RV-PA conduit & Patent & Patent & Reintervention \\
\hline
\end{tabular}

The final score is class 1 (optimal) if all subprocedure scores are optimal. The final score is class 2 (adequate) if any subprocedure score is adequate, but no subprocedure score is inadequate. The final score is class 3 (inadequate) if any subprocedure score is inadequate. NA, Not assessed in SVR trial core laboratory; MBTS, modified Blalock-Taussig shunt; $R V-P A$, right ventricle-to-pulmonary artery. 
TABLE E2. Patient and Norwood procedural characteristics

\begin{tabular}{|c|c|c|c|}
\hline Characteristic & Total (n) & Value & Median (IQR) \\
\hline \multicolumn{4}{|l|}{ Demographic data } \\
\hline Male sex & 549 & $340(62)$ & \\
\hline Birth weight $(\mathrm{kg})$ & 549 & $3.1 \pm 0.5$ & $3.1(2.8-3.5)$ \\
\hline Birth weight $<2.5 \mathrm{~kg}$ & 549 & $76(14)$ & \\
\hline Gestational age (wk) & 549 & $38.2 \pm 1.6$ & $38(37-39)$ \\
\hline Gestational age $<37 \mathrm{wk}$ & 549 & $64(12)$ & \\
\hline Hispanic & 539 & $101(19)$ & \\
\hline Race & 544 & & \\
\hline White & & $436(80)$ & \\
\hline Black & & $86(16)$ & \\
\hline Other & & $22(4)$ & \\
\hline 1-min Apgar (after imputation) & 549 & $7.6 \pm 1.6$ & $8(8-9)$ \\
\hline 5-min Apgar (after imputation) & 549 & $8.6 \pm 0.8$ & $9(8-9)$ \\
\hline \multicolumn{4}{|l|}{ Before Norwood } \\
\hline Prenatal diagnosis of CHD & 549 & $420(77)$ & \\
\hline Fetal intervention & 549 & $14(3)$ & \\
\hline HLHS & 549 & $474(86)$ & \\
\hline Aortic atresia & 549 & $343(63)$ & \\
\hline Obstructed pulmonary venous return & 549 & $19(4)$ & \\
\hline Highest lactate level (mmol/L; after imputation) & 549 & $3.9 \pm 2.9$ & $3.0(2.5-4.0)$ \\
\hline Ever intubated & 547 & $263(48)$ & \\
\hline For apnea & 547 & $75(14)$ & \\
\hline For shock & 547 & $14(3)$ & \\
\hline For respiratory failure & 547 & $72(13)$ & \\
\hline For metabolic acidosis & 547 & $31(6)$ & \\
\hline Cardiac and other surgery & 549 & & \\
\hline Yes & & $11(2)$ & \\
\hline No & & $538(98)$ & \\
\hline Cardiac catheterization intervention & 549 & & \\
\hline Yes & & $28(5)$ & \\
\hline No & & $521(95)$ & \\
\hline Complications & 549 & & \\
\hline Yes & & $153(28)$ & \\
\hline No & & $396(72)$ & \\
\hline No. of complications & & $0.6 \pm 1.2$ & $0(0-1)$ \\
\hline Age at Norwood procedure (d) & 549 & $5.8 \pm 4.1$ & $5(3-7)$ \\
\hline Weight at Norwood procedure $(\mathrm{kg})$ & 549 & $3.1 \pm 0.5$ & $3.2(2.8-3.5)$ \\
\hline \multicolumn{4}{|l|}{ Norwood hospitalization* } \\
\hline Crossclamp time (min) & 549 & $56.0 \pm 23.4$ & $53(40-67)$ \\
\hline Total support time (min) & 549 & $143.9 \pm 54.1$ & $139(105-171)$ \\
\hline Perfusion type & 544 & & \\
\hline DHCA & & $296(54)$ & \\
\hline $\mathrm{RCP}$ & & $130(24)$ & \\
\hline DHCA and RCP & & $118(22)$ & \\
\hline DHCA duration (min) & 544 & $31.7 \pm 23.2$ & $0(0-51)$ \\
\hline RCP duration (min) & 546 & $23.8 \pm 29.2$ & $0(0-117)$ \\
\hline Exterior diameter of AA at STJ (mm) & 535 & $3.12 \pm 1.66$ & $2.5(2.0-4.0)$ \\
\hline ICU total LOS (d) & 539 & $25.8 \pm 33.8$ & $14.0(9.0-28.0)$ \\
\hline Open sternum postoperatively & 544 & $426(78)$ & \\
\hline Pacemaker insertion at or after operation & 544 & $6(1)$ & \\
\hline Oxygen saturation at discharge $(\%)$ & 436 & $82.4 \pm 4.9$ & $82(80-85)$ \\
\hline
\end{tabular}

Data presented as n, n (\%), or mean \pm standard deviation, unless otherwise noted. IQR, Interquartile range; $C H D$, congenital heart disease; HLHS, hypoplastic left heart syndrome; $D H C A$, deep hypothermic circulatory arrest; $R C P$, regional cerebral perfusion; $A A$, ascending aorta; STJ, sinotubular junction; ICU, intensive care unit; $L O S$, length of stay. $*$ These postbaseline factors were not used as candidate predictors in the modeling of outcomes. 
TABLE E3. Baseline characteristics of patients with and without TPSs

\begin{tabular}{|c|c|c|c|c|}
\hline Characteristic & TPS assignable (class $1,2,3$ ) & Class 4* & Class 5 & $P$ value \\
\hline Patients (n) & 356 & 56 & 137 & \\
\hline Center & & & & $<.001 \ddagger$ \\
\hline \multicolumn{5}{|l|}{ Demographic data } \\
\hline Male sex & $216(61)$ & $29(52)$ & $95(69)$ & .05 \\
\hline Birth weight $(\mathrm{kg})$ & $3.1 \pm 0.5$ & $2.9 \pm 0.6$ & $3.2 \pm 0.5$ & $.02 \ddagger$ \\
\hline Birth weight $<2.5 \mathrm{~kg}$ & $44(12)$ & $17(30)$ & $15(11)$ & $<.001 \ddagger$ \\
\hline Gestational age (wk) & $38.2 \pm 1.6$ & $37.6 \pm 1.9$ & $38.3 \pm 1.5$ & $.03 \ddagger$ \\
\hline Gestational age $<37$ wk & $37(10)$ & $13(23)$ & $14(10)$ & $.02 \ddagger$ \\
\hline Hispanic & $74(21)$ & $5(9)$ & $22(16)$ & .09 \\
\hline Race & & & & .73 \\
\hline White & $287(82)$ & $43(77)$ & $106(78)$ & \\
\hline Black & $50(14)$ & $11(20)$ & $25(18)$ & \\
\hline Other & $15(4)$ & $2(4)$ & $5(4)$ & \\
\hline 1-min Apgar (after imputation) & $8.0(8.0-9.0)$ & $8.0(8.0-8.0)$ & $8.0(8.0-8.0)$ & .23 \\
\hline 5-min Apgar (after imputation) & $9.0(8.0-9.0)$ & $9.0(8.0-9.0)$ & $9.0(8.0-9.0)$ & .11 \\
\hline SES score & $0.1 \pm 5.1$ & $-0.8 \pm 5.9$ & $-0.3 \pm 4.6$ & .43 \\
\hline Below poverty level $(\%)$ & $12.6 \pm 12.6$ & $14.2 \pm 14.2$ & $13.2 \pm 12.0$ & .67 \\
\hline \multicolumn{5}{|l|}{ Pre-Norwood } \\
\hline Prenatal diagnosis of $\mathrm{CHD}$ & 274 (77) & $43(77)$ & $103(75)$ & .92 \\
\hline Fetal intervention & $8(2)$ & $0(0)$ & $6(4)$ & .18 \\
\hline HLHS & $307(86)$ & $44(79)$ & $123(90)$ & .12 \\
\hline Aortic atresia & $217(61)$ & $35(63)$ & $91(66)$ & .53 \\
\hline Obstructed pulmonary venous return & $8(2)$ & $4(7)$ & $7(5)$ & .08 \\
\hline Highest lactate level (mmol/L) & $3.9 \pm 2.9$ & $3.7 \pm 2.6$ & $3.9 \pm 3.0$ & .87 \\
\hline Ever intubated & $162(46)$ & $32(57)$ & $69(50)$ & .27 \\
\hline For apnea & $41(12)$ & $9(16)$ & $25(18)$ & .14 \\
\hline For shock & $9(3)$ & $3(5)$ & $2(2)$ & .30 \\
\hline For respiratory failure & $48(14)$ & $7(12)$ & $17(12)$ & .93 \\
\hline For metabolic acidosis & $21(6)$ & $3(5)$ & $7(5)$ & .93 \\
\hline Cardiac and other surgery & $7(2)$ & $0(0)$ & $4(3)$ & .42 \\
\hline Cardiac catheterization intervention & $18(5)$ & $3(5)$ & $7(5)$ & .99 \\
\hline Complications & $98(28)$ & $19(34)$ & $36(264)$ & .54 \\
\hline \multicolumn{5}{|l|}{ At Norwood operation } \\
\hline Age $(\mathrm{d})$ & $5.8 \pm 4.2$ & $5.5 \pm 3.5$ & $5.9 \pm 3.9$ & .82 \\
\hline Weight $(\mathrm{kg})$ & $3.1 \pm 0.5$ & $2.9 \pm 0.6$ & $3.1 \pm 0.5$ & $.004 \ddagger$ \\
\hline Weight-for-age z score (WHO) & $-0.6 \pm 1.2$ & $-1.2 \pm 1.3$ & $-0.6 \pm 1.1$ & $.010 \ddagger$ \\
\hline Exterior diameter of AA $(\mathrm{mm})$ & $3.1 \pm 1.6$ & $3.0 \pm 1.6$ & $3.2 \pm 1.8$ & .79 \\
\hline Genetic syndrome or other anomalies & & & & $<.001 \ddagger$ \\
\hline Yes & $78(22)$ & $6(11)$ & $35(25)$ & \\
\hline No & $175(49)$ & $7(13)$ & $73(53)$ & \\
\hline Unknown & $103(29)$ & $43(77)$ & $29(21)$ & \\
\hline
\end{tabular}

Data presented as $\mathrm{n}(\%)$, mean \pm standard deviation, or median (interquartile range). TPS, Technical performance score; SES, socioeconomic status; CHD, congenital heart disease; HLHS, hypoplastic left heart syndrome; WHO, World Health Organization; $A A$, ascending aorta. *No TPS assigned because no echocardiogram performed and no unplanned reinterventions required before discharge. †No TPS assigned because of an incomplete echocardiogram and no unplanned reinterventions required before discharge. $\ddagger$ Statistically significant. 
TABLE E4. Early mortality or transplantation rate stratified by TPS class

\begin{tabular}{|c|c|c|c|c|}
\hline Variable & Patients (n) & Transplantation or death (n) & Transplantation (n) & Death (n) \\
\hline Overall & 549 & $101(21)$ & 9 & 92 \\
\hline \multicolumn{5}{|l|}{ TPS } \\
\hline Class 1 (optimal) & 255 & $10(4)$ & 3 & 7 \\
\hline Class 2 (adequate) & 42 & $3(7)$ & 0 & 3 \\
\hline Class 3 (inadequate) & 59 & $16(27)$ & 2 & 14 \\
\hline Class 4 (no ECG, no RI) & 56 & $54(96)$ & 3 & 51 \\
\hline Class 5 (incomplete ECG, no RI) & 137 & $18(13)$ & 1 & 17 \\
\hline
\end{tabular}

Data in parentheses are percentages. TPS, Technical performance score; $E C G$, echocardiogram; RI, reintervention.

TABLE E5. Prestage II reintervention stratified by TPS

\begin{tabular}{lccc}
\hline \multicolumn{1}{c}{ Variable } & Patients (n) & Reintervention & OR $(\mathbf{9 5} \% \mathbf{C I})$ \\
\hline Overall & 329 & $77(23)$ & \\
TPS & & & \\
Class 1 (optimal) & 247 & $47(19)$ & $0.30(0.15-0.62)$ \\
Class 2 (adequate) & 39 & $17(44)$ & $1.78(0.72-4.42)$ \\
Class 3 (inadequate) & 43 & $13(30)$ & Reference \\
\hline
\end{tabular}

Data presented as n (\%), unless otherwise noted. TPS, Technical performance score; $O R$, odds ratio; $C I$, confidence interval. *Statistically significant.

TABLE E6. Late mortality or transplantation from Norwood discharge to 3 years after randomization

\begin{tabular}{|c|c|c|c|c|}
\hline Variable & Patients (n) & Transplantation or death (n) & Transplantation (n) & Death (n) \\
\hline Overall & 448 & $96(21)$ & 11 & 85 \\
\hline \multicolumn{5}{|l|}{ TPS } \\
\hline Class 1 (optimal) & 245 & $55(55 / 242[23])^{*}$ & 6 & 49 \\
\hline Class 2 (adequate) & 39 & $5(5 / 38[13]) *$ & 1 & 4 \\
\hline Class 3 (inadequate) & 43 & $9(9 / 42[21])^{*}$ & 2 & 7 \\
\hline Class 4 (no ECG) & 2 & $2(100)$ & 0 & 2 \\
\hline Class 5 (incomplete ECG) & 119 & $25(26)$ & 2 & 23 \\
\hline
\end{tabular}

Data in parentheses or brackets are percentages. The data of the subjects who died or underwent cardiac transplantation before Norwood discharge were excluded. TPS, Technical performance score; $E C G$, echocardiogram. *The denominators reflect the exclusion of the 5 patients who withdrew 13 to 16 months after randomization.

TABLE E7. Multivariable Cox regression model for late mortality or transplantation from Norwood discharge to 3 years after randomization $(\mathbf{n}=\mathbf{3 2 7})$

\begin{tabular}{lccc}
\hline \multicolumn{1}{c}{ Variable } & HR & $\mathbf{9 5} \%$ CI & P value \\
\hline Gestational age $<37 \mathrm{wk}$ & & & $.010^{*}$ \\
$\quad$ Yes & 2.32 & $1.23-4.41$ & \\
$\quad$ No & Reference & & \\
Obstructed pulmonary venous return & 5.43 & $1.66-17.8$ & $.005^{*}$ \\
Highest lactate level (mmol/L) & 1.11 & $1.05-1.18$ & $<.001^{*}$ \\
TPS & & & .390 \\
Class 1 (optimal) & 1.37 & $0.67-2.82$ & \\
Class 2 (adequate) & 0.80 & $0.26-2.45$ & \\
Class 3 (inadequate) & Reference & & \\
\hline
\end{tabular}

The data of subjects who died or underwent cardiac transplant before Norwood discharge were excluded. $H R$, Hazard ratio; $C I$, confidence interval; TPS, technical performance score. *Statistically significant.
TABLE E8. Types of unplanned reinterventions in anatomic areas of surgical repair during Norwood hospitalization in class 3 patients stratified by shunt type

\begin{tabular}{lcc}
\hline Unplanned reintervention & BTS (n) & RVPAS (n) \\
\hline Shunt & 20 & 24 \\
$\quad$ Surgical thrombectomy & 2 & 0 \\
Catheter RI & 6 & 10 \\
Shunt crossover & 5 & 7 \\
Surgical revision & 7 & 7 \\
Arch & 3 & 1 \\
Branch PA & 4 & 2 \\
ASD & 1 & 0 \\
Total & 28 & 27 \\
\hline
\end{tabular}

For the entire cohort of 55 patients, 9 patients had no ECGs and 13 had incomplete ECGs. BTS, Blalock-Taussig shunt; RVPAS, right ventricle-to-pulmonary artery shunt; $R I$, reintervention; $P A$, pulmonary artery; $A S D$, atrial septal defect. 
TABLE E9. Echocardiographic findings of 59 class 3 patients

\begin{tabular}{|c|c|c|c|}
\hline $\begin{array}{c}\text { Arch peak } \\
\text { velocity }(\mathbf{m} / \mathbf{s})\end{array}$ & $\begin{array}{c}\text { ASD mean } \\
\text { gradient }(\mathrm{mm} \mathrm{Hg})\end{array}$ & RI & Patients (n) \\
\hline$<2.25^{*}$ & $<2$ & Proximal arch & 1 \\
\hline $2.25-3.2 \dagger$ & $<2$ & Proximal arch & 1 \\
\hline Missing & Missing & Proximal arch & 1 \\
\hline $2.25-3.2$ & $>4$ & Atrial septectomy & 1 \\
\hline$<2.25$ & $>4$ & $\begin{array}{l}\text { Atrial septectomy } \\
\text { and RI }\end{array}$ & 1 \\
\hline Missing & Missing & Shunt RI & 8 \\
\hline Missing & $<2$ & Shunt RI & 13 \\
\hline$<2.25$ & $<2$ & Shunt RI & 27 \\
\hline $2.25-3.2$ & Missing & Shunt RI & 1 \\
\hline $2.25-3.2$ & $<2$ & Shunt RI & 1 \\
\hline$<2.25$ & $>4$ & None & 1 \\
\hline$>3.2 \ddagger$ & $<2$ & None & 3 \\
\hline
\end{tabular}

